\title{
Background-Foreground Segmentation Based on Dominant Motion Estimation and Static Segmentation
}

\author{
Yu Huang, Dietrich Paulus, Heinrich Niemann \\ Chair for Pattern Recognition, Dept. of Computer Science University of Erlangen-Nürnberg, Erlangen, Germany
}

\begin{abstract}
This paper addresses the problem of image segmentation using motion and luminance information. We use the dominant motion model to calculate both the background and foreground motion in a robust estimation framework and then combine it with the result of static segmentation using the watershed algorithm to segment the foreground from the background. In this paper, the previous pixelbased (or over a small neighborhood) motion measure is replaced by the patch-based motion measure in motion segmentation. Experimental results are given to show the efficiency of our method.
\end{abstract}

Keywords: dominant motion, robust estimation, static segmentation, watershed

\section{Introduction}

The segmentation of image sequences into regions or 'objects' has received a large attention in recent years. Applications like object tracking, video coding and structure from motion can benefit from a meaningful segmentation. But it is by now not solved being a chicken-and-egg problem.

The methods of motion segmentation can be grouped into two broad classes (Sawhney, 1996). One class solves the problem by letting multiple models simultaneously compete for the description of the individual motion measurements (Wang, 1994), and the other excavates the multiple models sequentially by solving for a dominant model (Irani, 1994). For the former method, difficulties occur at determination of the number of models or uncertainty of mixture models. The latter may confront puzzles in the case of absence of dominant motion, and it yet lacks competition amongst the motion models.
In this paper, we discuss the dominant motionbased method used for background and foreground segmentation. In Sect. 2, we present related works and background. In Sect. 3, the dominant motion estimation method described in (Black, 1996) is outlined, and its combination with static segmentation using the watershed algorithm is presented. Finally experimental results are reported in Sect. 4 and concluding remarks are given in Sect. 5.

\section{Background}

The dominant motion model-based method used for segmention, compared to the multiple model competition method, is more efficient because it does not need to consider how many objects occur in the scene and looks simpler from its algorithmic form. It is valid for some application fields, for example, background/foreground segmentation.

In the use of dominant motion model, one of the key steps is determination of the dominant object. It is a region or object corresponding to the dominant motion. (Black, 1996) put forward a dominant motion estimation method in a simulated annealing framework, but it cannot give a clean region segmentation since the motion measure of each pixel is individually computed only. (Irani, 1994) also use the dominant motion model for segmentation; they give a motion measure based on the weighted average of the normal flow magnitudes over a small neighborhood. Its shortcomings are the lack of determination of the meaningful regions or patches. 


\section{Patch-based dominant motion segmentation}

In this paper we combine the static segmentation with the dominant motion model. Here oversegmentation is needed in the process of static segmentation in order to make pixels in each subregion having the similar motion. There are some current methods available for this task, for example, the watershed algorithm, the pixelbased region growing and the quadtree splitmerge method etc. In this paper, we choose the watershed algorithm. Based on the static segmentation result, we replace the pixel-based motion measure with the proposed patch or region-based motion measure to make a clear segmentation of the dominant motion region.

\subsection{The SOR method for dominant motion estimation}

Before we present our approach, the Simultaneous-Over-Relaxation (SOR) method (Black, 1996) for dominant motion estimation is described simply. First, the interframe motion is defined as

$$
f(\mathbf{x}, t+1)=f(\mathbf{x}-\mathbf{u}(\mathbf{x} ; \mathbf{a}), t),
$$

where $f(\mathbf{x}, t)$ is the brightness function in time instant $t, \mathbf{x}=(x, y)$ is coordinate of the image pixel, and $\mathbf{u}(\mathbf{x} ; \mathbf{a})$ is the motion vector. We assume the affine flow model ( 6 parameters) for the dominant object

$$
\mathbf{u}(\mathbf{x} ; \mathbf{a})=\left[\begin{array}{l}
u(x, y) \\
v(x, y)
\end{array}\right]=\left[\begin{array}{l}
a_{0}+a_{1} x+a_{2} y \\
a_{3}+a_{4} x+a_{5} y
\end{array}\right]
$$

where $\mathbf{a}=\left(a_{0}, a_{1}, a_{2}, a_{3}, a_{4}, a_{5}\right)^{\mathrm{T}}$ are the parameters of the affine model. This model is valid when the depth variance is small enough compared with the depth from the camera. Dominant motion estimation is formulated as the following robust $\mathrm{M}$-estimator,

$$
\min _{u, v} E_{D}=\sum_{(x, y) \in \mathbf{R}} \rho\left(u f_{x}+v f_{y}+f_{t}, \sigma\right)
$$

here the $\rho$ - function is chosen as the GemanMcClure function (Black, 1996),

$$
\rho(x, \sigma)=\frac{x^{2}}{x^{2}+\sigma^{2}},
$$

with $\sigma$ as the scale parameter, and $f_{x}, f_{y}, f_{t}$ as partial derivatives of brightness function with respect to $x, y$ and $t$. The SOR iteration update equations are

$$
a_{i}^{(n+1)}=a_{i}^{(n)}-\omega \frac{\partial E_{D}}{T_{a_{i}} \partial a_{i}}
$$

with $\omega=1.995(0<\omega<2), T_{a_{i}}$ as the upper bound of the second-order partial derivatives, $i$. e.

$$
T_{a_{i}} \geq \frac{\partial^{2} E_{D}}{\partial a_{i}^{2}} .
$$

The algorithm begins by constructing the Gaussian pyramid (we make three levels). At the coarse level motion is initially set to zero. The number of iterations is chosen as 10 . When the estimated parameters are interpolated into the next level, these parameters are used to warp the first image to the second one. In the current level only the change in the paramenters is estimated in the iterative update scheme.

The SOR method lowers the scale parameter $\sigma$ according to the formula $\sigma_{n+1}=0.95 \sigma_{n}$. The effect is similar to the simulated annealing method. We set initially $\sigma$ as $25 \sqrt{3}$ and finally $\sigma$ (its lower bound) as $15 \sqrt{3}$. Once the dominant motion is estimated the outlying measurements are determined by checking the value $\left|f_{x} u+f_{y} v+f_{t}\right| \geq \tau$, here $\tau=\sigma / \sqrt{3}$. These outlier pixels can be used to determine the next dominant motion parameters.

\subsection{Watershed technique of static segmentation}

The watershed technique is one of the classics in the field of topography. It regards the gradient magnitude image as a landscape where the brightness values correspond to the elevation. Areas where a rain drop would drain to the same minimum are denoted as catchment basins, and the lines separating adjacent catchment basins are called dividing lines or watersheds.

We obtain the watersheds of the gradient image applying the method in (Vincent, 1991) proposed by Vincent and Soille. The operation of their technique can simply be described by figuring that holes are pierced in each local minimum of the topographic relief. At the end, the surface is slowly immersed into a 'lake', 
filling all the catchment basins, starting from the basin that is associated to the global minimum. While two catchment basins tend to merge, a dam is built. The process results in partitioning of the image in many catchment basins, of which the borders define the watersheds. A severe drawback to the computation of watershed images is oversegmentation. Here, though, we need oversegmentation in this procedure, but too small partitioned subregions will reduce the accuracy of the following motion labeling process, especially on those poor textured regions. (Vincent, 1991) suggests modifying the gradient function so that the resulting catchment basins correspond only to the desired objects. They put forward two types of methods to realize it, one is region growing, another is utilization of some knowledges on the images studied. Here we use: 1. Prefiltering (mean value filtering in the $3 \times 3$ neighborhoods) to allevaite the random noise; 2. After finding the watersheds, the adjacent catchment basins (4-neighborhoods searching) are further iteratively merged (normally the number of iterations is 10) based on thresholding the difference between two adjacent subregions' mean values.

\subsection{Motion measure}

We use the static segmentation to get small regions and then determine each region's motion measure from MAE (Mean Absolute Error) of difference between the warped image and the origin image, i. e.

$$
M_{i, j}=\sum_{\mathbf{x} \in \mathbf{R}_{i}}\left|f(\mathbf{x}, t+1)-f^{W_{j}}(\mathbf{x}, t)\right| / C_{i},
$$

where $f^{W_{j}}(\mathbf{x}, t)$ is the warped image of $f(\mathbf{x}, t)$ using the $j$ th dominant motion parameters, $C_{i}$ is the pixel number in the subregion $\mathbf{R}_{i}$. If we only consider the two dominant motion models in the scene, like the background and foreground segmentation, we set $j$ as 2 , as for $i=1,2, \ldots N$ ( $N$ is the number of regions after static segmentation).

We make the segmentation of the second frame because the motion measure is calculated from its difference with the warped frame. Here we consider two motion models, so directly comparing the results of (7) and choosing the motion label $j$ corresponding to the minimal one. Then, we can segment the foreground from background. If the background is static, i. e. the camera is not moving, we can realize the moving detection too.

\section{Results of experiments}

We realize the method in C on a SGI workstation. We did experiments with different image sequences, mainly considering whether the camera was moving or not. The segmentation from two consecutive images required about 35 seconds, half of time is for static segmentation.

\subsection{Static camera}

First we give results from two consecutive images in a gait sequence, shown in Fig. 1(a) and (b). The camera is static, and the person in the corridor just begins to walk. Image size is 384x256. Fig. 1(c) and (d) shows the watershed segmentation results (with the overlapped region boundaries) before and after merging adjecent regions. Fig. 1(e) and (f) give the foreground segmentation results with the method in (Black, 1996) and our proposed method on the same input images respectively. Although in Fig. 1(e) it locates the foreground but cannot output a clear region. In Fig. 1(f), there exists some errors in the upper left and right corners of images, they are caused by the calculation error of the motion measure in those subregions warped out of the image. The estimated affine motion parameters are in table 1.

\subsection{Moving camera and moving objects}

Then the results from two consecutive frames of the standard MPEG 'Coast Guard' image sequence in Fig. 2(a) and (b) are given too, in which a small boat is moving while the camera is panning. Image size is $352 \times 240$. The figures' location arrangement of Fig. 2 is the same as Fig. 1. From Fig. 2(e) we can find that the result under natural outdoors scenes using the method in (Black, 1996) can not group coherent moving pixels to a connected region. The estimated affine motion parameters are in table 2. 


\begin{tabular}{|c|c|c|c|c|c|c|}
\hline & $\mathrm{a}[1]$ & $\mathrm{a}[2]$ & $\mathrm{a}[3]$ & $\mathrm{a}[4]$ & $\mathrm{a}[5]$ & \\
\hline \hline Foreground & 5.176360 & 0.013673 & 0.025182 & 2.629096 & 0.012113 & 0.001330 \\
\hline Background & 0.197737 & -0.000941 & 0.000573 & 0.010358 & -0.000561 & 0.001848 \\
\hline
\end{tabular}

Table 1. Affine parameters in "gait" images.

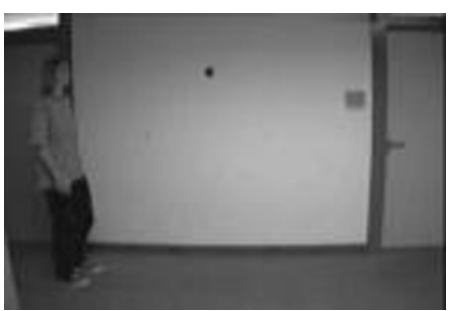

(a) first frame

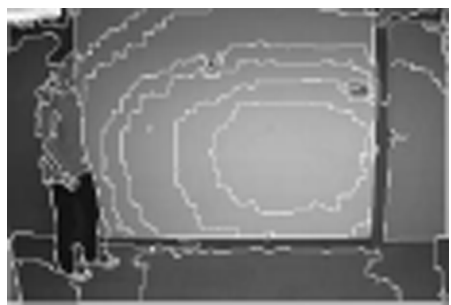

(d) watershed segmentation after merging

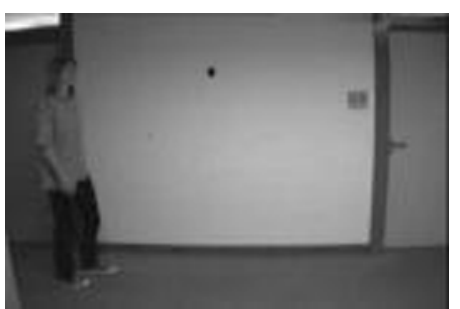

(b) second frame

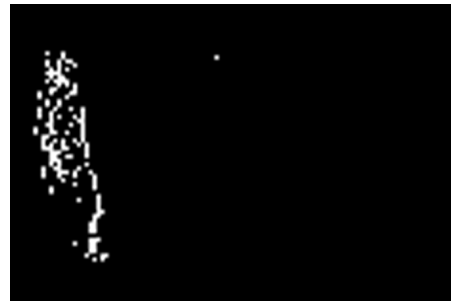

(e) dominant (white) pixels

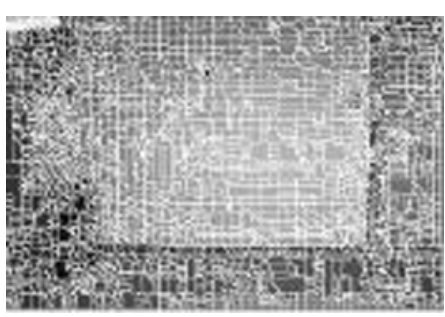

(c) watershed segmentation before merging

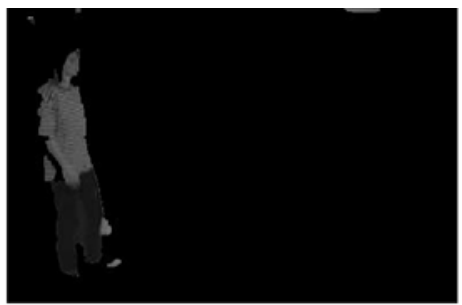

(f) dominant motion segmentation

Fig. 1. Gait sequence.

\begin{tabular}{|c|c|c|c|c|c|c|}
\hline & $\mathrm{a}[0]$ & $\mathrm{a}[1]$ & $\mathrm{a}[2]$ & $\mathrm{a}[3]$ & $\mathrm{a}[4]$ & $\mathrm{a}[5]$ \\
\hline \hline Foreground & -0.513183 & 0.002020 & -0.007339 & -0.124831 & -0.003332 & 0.019044 \\
\hline Background & 0.907186 & -0.001687 & -0.004894 & 0.066219 & -0.000159 & 0.000222 \\
\hline
\end{tabular}

Table 2. Affine parameters in "Coast guard" images.

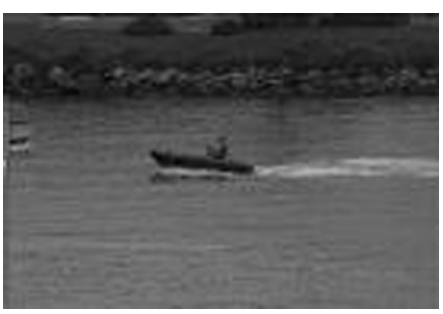

(a) first frame

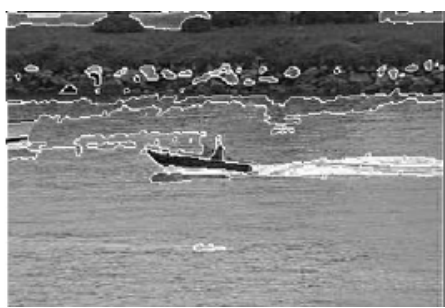

(d) watershed segmentation after merging

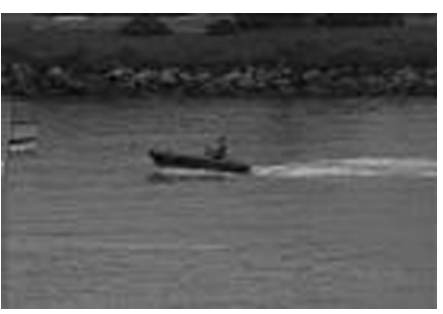

(b) second frame

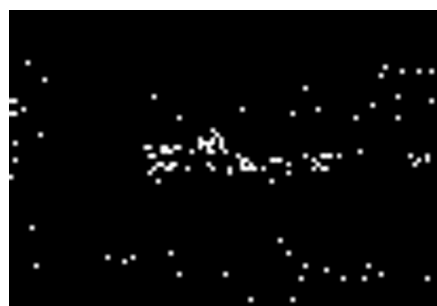

(e) dominant (white) pixels

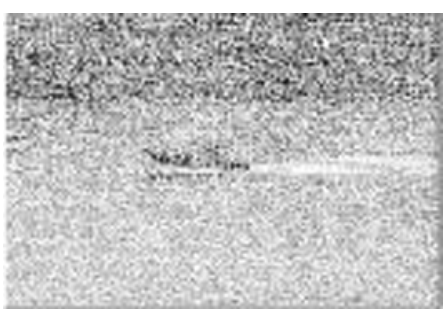

(c) watershed segmentation before merging

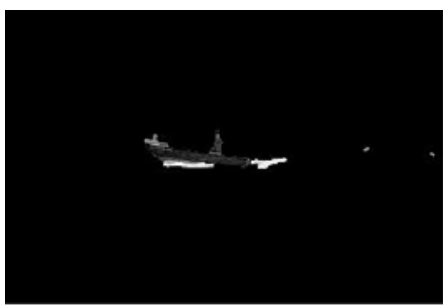

(f) dominant motion segmentation

Fig. 2. Coast guard sequence. 


\section{Conclusion}

We report a motion segmentation method, which combined the static segmentation using the watershed algorithm and the dominant motion model. We replace the pixel-based motion measure with the patch-based motion measure. From given experiment results, we show the method efficiency. In future, we will consider the temporal coherence or motion prediction in the motion segmentation from the entire sequence. Meanwhile, we will test its validity in the tasks of posture or gesture recognition.

\section{Acknowledgement}

This research is partially supported by Alexander von Humboldt foundation.

\section{References}

[1] BLACK M. J. (1996), The robust estimation of multiple motions: parametric and piecewise-smooth flow fields, $C V \& I P, 63(1): 75-104$.

[2] IRANI M. (1994), Computing occluding and transparent motions, Int. J. Computer Vision, 12(1): 5-16.

[3] SAWhnEy H. (1996), Compact representations of videos through dominant and multiple motion estimation, IEEE T-PAMI, 18(8): 814-830.

[4] VINCENT L. (1991), Watersheds in digital spaces: a efficient algorithm based on immersion simulations, IEEE T-PAMI, 13(6): 583-589.

[5] WANG J. (1994), Representing moving images with layers, IEEE T-IP, 3(5): 625-638.

Received: October, 2000 Accepted: November, 2000

Contact address: Yu Huang Dietrich Paulus Heinrich Nieman Chair for Pattern Recognition Dept. of Computer Science University of Erlangen-Nürnberg 91058, Erlangen, Germany
DR. YU HUANG was born in the PR China. He got his B.S. Degree in 1990 at the Dept. of Information \& Control Engineering, Xi' an Jiaotong University, Xi'an city, PR China, M.S. degree in 1993 at the Department of Electrical Engineering, Xidian University, Xian city, PR China, and $\mathrm{PhD}$ degree in engineering in 1997 at the Institute of Information Science, Northern Jiaotong University, Beijing, PR China. From April 1997 to April 1999, he was assistant professor and postdoctoral fellow at the Dept. of Computer Science \& Technology, Tsinghua University, Beijing, PR China. In Sept. 1999 he entered Chair for Pattern Recognition, University of Erlangen-Nïrnberg, Erlangen, Germany, as a research fellow of Prof. H. Niemann, supported by Alexander von Humboldt Foundation. His interests include motion-based segmentation, video indexing, vision-based HCI and Augmented reality.

DR.-ING. DIETRICH PAULUS received his Bachelor Degree in computer science from the University of Western Ontario, London, Canada (1983). He graduated (1987) and received his PhD Degree (1991) and Habilitation (2000) at Universiät Erlangen-Nürnberg, Germany. He is presently a senior reseacher (Akademischer Oberrat) in the field of image pattern recognition and is head of the vision group. He teaches courses in computer vision and applied programming for image processing. He has written and edited several books and proceedings, in particular on pattern recognition and image processing in $\mathrm{C}++$.

HEINRICH NIEMANN received the B.Sc. degree in electrical engineering and the $\mathrm{PhD}$ degree from Technical University Hannover in 1966 and 1969, respectively. During 1966/67 he was a graduate student at the University of Illinois, Urbana. From 1967 to 1972 he was with Fraunhofer Institut für Informationsverarbeitung in Technik und Biologie, Karlsruhe, working in the field of pattern recognition and biological cybernetics. During 1973 - 1975 he was teaching at Fachhochschule Giessen in the department of Electrical Engineering. Since 1975 he has been Professor of Computer Science at the University of ErlangenNirnberg, since 1988 he is also head of the research group 'Knowledge Processing' at the Bavarian Research Institute for Knowledge Based Systems (FORWISS) where he also was on the board of directors for six years. During 1979 - 1981 he was dean of the Engineering Faculty of the University.

His fields of research are speech and image understanding and the application of artificial intelligence techniques in these fields. Among other positions he was the Program Chairman at the International Conference on Pattern Recognition in 1982 and of its 'Computer Vision and Applications' track in 1992 and Program Co-Chairman at the International Conference on Acoustics, Speech, and Signal Processing in 1997. He is on the editorial board of Signal Processing, Pattern Recognition Letters, Pattern Recognition and Image Analysis, Journal of Computing and Information Technology, and Computers and Electrical Engineering. He is the author or coauthor of 6 books and about 300 journal and conference contributions as well as the editor or coeditor of 24 proceedings and special issues. He is a member of DAGM, ESCA, EURASIP, GI, IEEE, and VDE. 